\title{
IMAGE QUALITY ASSESSMENT BASED ON WAVE ATOMS TRANSFORM
}

\author{
Zehira HADDAD ${ }^{1,2}$, Azeddine BEGHDADI ${ }^{1}$, Amina SERIR ${ }^{2}$, Anissa MOKRAOUI \\ ${ }^{1}$ L2TI, institut Galilée, université Paris 13, France \\ ${ }^{2}$ LTIR, Faculté d'électronique et d'informatique, USTHB, Algérie
}

\begin{abstract}
Image quality assessment is still an active field of research. The main objective of the developed image quality metric is to offer an index of quality that is consistent with the human subjective judgment of image quality. Despite the great number of developed metrics, there is still a need for image analysis tools that is able to extract the most perceptual relevant characteristics of an image. The goal of this work is then to propose a more advanced analysis and representation tools to extract more effective features that could be incorporated in the design of the image quality metric. In this paper, we propose a novel objective metric based on wave atoms transform. This new transform is half multiscale and half multi-directional. It offers a better representation of images containing oscillatory patterns and textures than the others known transforms [10]. In this work, we propose a new full reference image quality metric based on wave atom transform and exploiting some properties of the human visual system. The consistency of the proposed metric with subjective evaluation is performed on LIVE database. The obtained correlation of this metric with the MOS provided by the database is better than other known metrics confirming thus the efficiency of this new image quality measure in predicting image quality.
\end{abstract}

Index Terms - Image quality assessment (IQA), Human visual system (HVS), Wave atoms transform.

\section{INTRODUCTION}

With the continuing development and improvement of image and video acquisition and processing systems, image quality assessment has gained much interest during these last decades. At the same time customers, i.e. observers, have become more and more critical, not only about the image resolution but also about the level of quality. Thus, the development of a method or a system to enable accurate assessment of the image quality becomes one of the most challenging research area that could significantly impact the performance of image and video processing applications. The ultimate goal of such research is to develop an objective quality assessment measure that could replace the subjective human evaluation.
Image quality assessment methods can be broadly classified into two categories, subjective and objective. Subjective methods are based on the human perceptual judgment. Whereas, in the second category, objective image quality metrics are derived from the observed images. For each category, three classes could be defined: full reference methods (FR) [1],[3], No reference methods (NR) and reduced reference methods (RR). For FR methods, both the original and distorted images are available for the computation of the image quality metric. Whereas, RR methods require only a set of features extracted from the original and the degraded images. NR methods, or blind methods, are used when only the degraded, or observed, image is available. For many practical applications, no reference (NR) methods are required.

In the case of FR methods, the most widely used objective image quality/distortion metrics is the Mean Square Error (MSE) and peak signal-to-noise ratio PSNR. However, it has been shown that these metrics are very often inconsistent with perceptual evaluation of image quality.

To overcome these limitations, many other metrics have been developed. The structural similarity metric [1] attracts a lot of attention for its first putting forward the concept of image structure feature and the assumption that human visual perception is highly adaptive for extracting structural features from natural scene. Yang et al investigate the feasibility of discrete wavelet transform based on structural similarity method [2]. H. R. Sheikh et al introduced information theory and put forwards information fidelity criterion [3] and visual information fidelity [4], in which image perceptual reduction is scored by rating the mutual information between the original and the distorted images. Chandle et al [5] proposed visual signal to noise ratio metric, dividing image distortion into near threshold and suprathreshold level, evaluates visual fidelity of image integrating the low level visual property of perceived contrast and the mid-level of global precedence.

Research on the primary visual cortex reveals that the Neuron cells in the V1 zone could be described by linear localized, oriented and bandpass filters [6]. These results suggest that for a computational image representation to be efficient, it should be based on a local, directional and multiresolution expansion. Pang et al [7] proposed a new objective quality metric based on directional information of 
image by projecting the image with radon transform. Gao et al [8] proposed an image quality metric using wavelet based contourlet transform. Contrast sensitivity function and a defined rational sensitivity threshold are applied to deal with the properties of HVS. Liu et al [9] proposed image quality assessment using contourlet transform. The distortion from different vision scales and edge directions is extracted by the image decomposition and a special masking based on Daly's model to take into account the human sensitivity system.

The aim of this work is to propose a more advanced analysis and representation tools (multidirectional and multiscale) to extract more effective features that could be incorporated in the derivation of the image quality metric. Among those tools, wave atom transform [10] is one of the most promising ones. Wave atom transform can be used to represent and analyse different types of images. This transform performs well especially in the representation of images containing anisotropic and oscillatory textures [10]. Images which contain this type of caracteristics are very difficult to analyse and represent. This specifity of wave atoms transform makes it very efficient for analyzing fine characteristics of images. Wave atoms transform appears as a promising tool for image distortion analysis.

This paper is organized as follows; in section 2 wave atoms transform is briefly introduced. The proposed wave atoms based image quality evaluation metric is introduced in section 3. Section 4 shows experimental results with comparison and analysis. Finaly, section 5 is devoted to conclusion and perspectives.

\section{WAVE ATOMS TRANSFORM}

Wavelet transform has been widely used to extract the image quality feature, emulating the multiresolution and localization properties of HVS [19]. However, it cannot effectively represent textures and fine details in images for lacking of directionality. Indeed, wavelets are not suited to the representation of anisotropic objects. Recently, a new theroy called multiscale geometric analysis has been developped (MGA) [11]. This has given rise to many transforms combining multiscale and multidirectional properties. Among these approaches, we find: Ridgelets [12], curvelets [13], contourlets [9], complex wavelets [18], cortex transform [14] and steerable pyramid. Wave atoms transform is also one of these approaches.

The main properties of wave atoms transform are the ability to adapt to arbitrary local directions of a pattern, i.e., warpings; and the ability to sparsely represent anisotropic patterns aligned with the axes [10].

In order to understand and situate wave atom transform among the other existing transforms, we use two parameters $\alpha$ and $\beta$ to index a lot of known wave packets architectures [10]. This description will clarify the connections between various transforms of modern harmonic analysis. The index $\alpha$ indicates whether the decomposition is multiscale $(\alpha=1)$ or not $(\alpha=0)$; and $\beta$ indicates whether basis elements are localized and poorly directional $(\beta=1)$ or, on the contrary, extended and fully directional $(\beta=0)$. This classification permits to classify, wavelets to $\alpha=\beta=1$. Ridgelet transform[12] will correspond to $\alpha=1$, $\beta=0$. For Gabor transform $\alpha=\beta=0$ and for curvelet transform $\alpha=1, \beta=1 / 2$ [13]. Wave atoms are defined for $\alpha=\beta=1 / 2$. Figure 1 illustrates this classification [10].

Wave atoms is noted as $\varphi_{\mu}(x)$, with subscript $\mu \stackrel{\Delta}{=}(j, m, n) \stackrel{\Delta}{=}\left(j, m_{1}, m_{2}, n_{1}, n_{2}\right)$. The indexes $j, m_{1}, m_{2}, n_{1}, n_{2}$ are integer-valued associated to a point $\left(x_{\mu}, y_{\mu}\right)$ in the phase-space defined as follows:

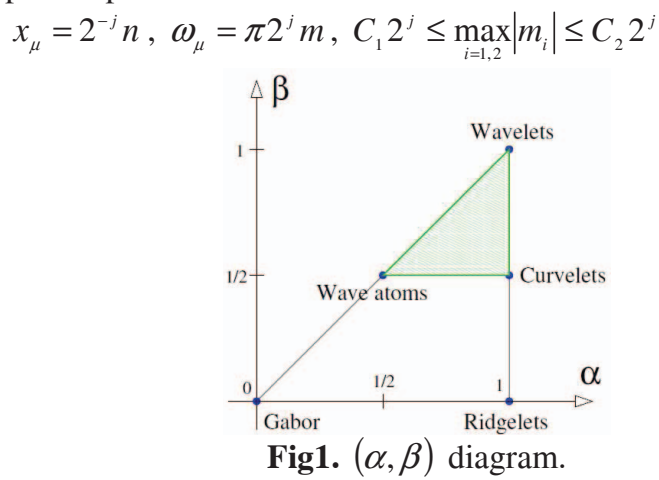

To better undertand wave atom in $2 \mathrm{D}$ case, let us adress first the $1 \mathrm{D}$ case. Wave atoms are constructed from tensor products of adequately chosen $1 \mathrm{D}$ wavelet packets. Onedimensional family of real-valued wave packets $\Psi_{m, n}^{j}(x), j \geq 0, m \geq 0, n \in \mathrm{Z}$, centered in frequency around $\pm \omega_{j, m}= \pm \pi 2^{j} m$, with $C_{1} 2^{j} \leq m \leq C_{2} 2^{j}$; and centered in space around $x_{j, n}=2^{-j} n$, is constructed. The onedimensional version of the parabolic scaling informs that the support of $\Psi_{m, n}^{j}(\omega)$ be of length $O\left(2^{2 j}\right)$, while $\omega_{j, m}=O\left(2^{2 j}\right)$ [10]. The bottom of figure 2-a illustrates the desired corresponding tiling of frequency. It is possible to construct an orthonormal basis satisfying these localization properties by using of a filter bank-based wavelet packet.

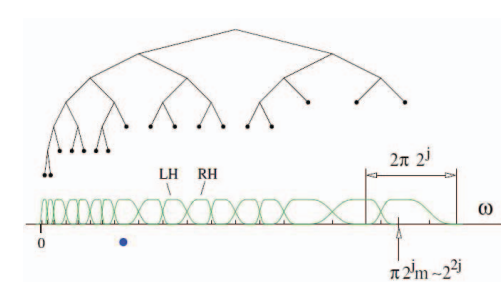

(a)

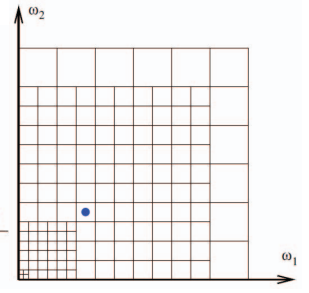

(b)
Fig2. (a) Wavelet-packet tree corresponding to wave atoms. (b) The wave atoms tiling of the frequency plane.

Figure 2-a presents the wavelet packet corresponding to wave atoms, defining the partitioning of the frequency axis 
in $1 \mathrm{D}$. It can be chosen to have depth $\mathrm{j}$ when the frequency is $2^{2 j}$. The bottom graph depicts Villemoes wavelet packets on the positive frequency axis. The frequency where a change of scale occurs is indicated by the dot under the axis. In this figure, "LH" indicates a left-handed window and "RH" indicates a right-handed window [10].

The construction presented above can be extended to $2 \mathrm{D}$ case. One may want to work with the original orthonormal basis $\varphi_{\mu}^{+}(x)$ instead of a tight frame. Since $\varphi_{\mu}^{+}(x)=\varphi_{\mu}^{(1)}(x)+\varphi_{\mu}^{(2)(x)}$, each basis function $\varphi_{\mu}^{+}(x)$ oscillates in two distinct directions, instead of one. This is the principle of orthobasis variant [10].

Figure 2-b represents the wave atom tiling of the special frequency plane. When the scale $\mathrm{j}$ increases by 1 , the squares size doubles. At a given scale $\mathrm{j}$, squares are indexed by $m_{1}, m_{2}$ starting from zero near the axes. The dot indicates the same change of scale as in Figure 2-a [10].

\section{THE PROPOSED IMAGE QUALITY METRIC}

Digital images are generally highly structured. A good image quality metric should be able to capture the key structural features. Wave atoms transform is a powerfull image representation tool. Wave atoms transform is efficient especially for images containing textures and oscillatory patterns. Wave atoms transform appears as a good candidate for evaluating image distortions. In order to make the proposed metric consistent with subjective evaluation appears, some properties of human visual system (HVS) should be taken into account in the design of this metric. The different steps of the proposed image quality metric are the following:

First, the original image $I_{R}$ and the distorted image $I_{D}$ are decomposed by wave atoms transform. We obtain the different wave atoms coefficients $c_{l, o}^{R}(x, y)$ and $c_{l, o}^{D}(x, y)$ corresponding at site $(x, y)$ in the subband $(l, o)$ respectively for the images $I_{R}$ and $I_{D}$.

After this, we apply a HVS based masking effect. Knowing that the visibility of one image component (the target) is reduced by the presence of another (the background). Watson et al showed that image quality models should incorporate entropy masking, as well as contrast masking [15]. In order to take into account the modification of the visibility threshold due to the contrast value, we use a contrast making. Furthermore, in order to consider the visibility threshold modification due to the neighborhood characteristic, an entropy masking is used.

We apply here, a semi-local masking inspired by Daly's model [16]. We calculate for each site $(x, y)$ at the scale $l$ and the orientation $o$, the following visibility threshold elevation $T_{l, o}(x, y)$ :
$T_{l, o}(x, y)=\left(1+\left(k_{1} \cdot\left(k_{2} \cdot\left|c_{l, o}(x, y)\right|\right)^{S+\Delta s(x, y)}\right)^{b}\right)^{1 / b}$

$k_{1}, k_{2}$ correspond to the pivot point of the contrast curve. $b$ determines how closely the curve follow the asymptote in the transition region. $s(x, y)=S+\Delta s(x, y)$ represents the slope of the high masking contrast asymptote, depending on the neighborhood. $\Delta s(x, y)$ is calculated from the entropy on a neighborhood of the tested image:

$\Delta s(x, y)=\frac{b_{1}}{1+e^{-b_{2}\left(E(x, y)-b_{3}\right)}}$
$E(x, y)=-\sum p(x, y) \log (p(x, y))$

$E(x, y)$ is the resulting entropy map calculated from the probability $p(x, y)$ deducted from the luminance histogram of neighborhood around site $(x, y) . b_{1}, b_{2}, b_{3}$ are adjustable parameters. After different tests to adjust these parameters, we finally used the following values:

$b_{1}=0.3 ; b_{2}=2 ; b_{3}=1 ; k_{1}=k_{2}=1 ; b=2 ; S=0.65$

After this step, we normalize the error between the wave atoms coefficients of the original image and the distorted image by the following equation:

$N E_{l, o}(x, y)=\frac{\left|c_{l, o}^{R}-c_{l, o}^{D}\right|}{\max \left(T_{l, o}^{R}(x, y), T_{l, o}^{D}(x, y)\right)}$

To achieve a single value representing image quality, we must combine the different informations from each location, scale and orientation subband.

$$
\begin{aligned}
& N E_{l, o}=\left(\frac{1}{X_{l, o} \cdot Y_{l, o}} \sum_{x=1}^{X_{l, o}} \sum_{y=1}^{Y_{l, o}}\left(N E_{l, o}(x, y)\right)^{2}\right)^{1 / 2} \\
& N E=\frac{1}{L} \sum_{l=1}^{L}\left(\frac{1}{O_{l}} \sum_{o=1}^{O_{l}} N E_{l, o}\right)
\end{aligned}
$$

Finally, the proposed wave atoms based image quality evaluation metric is derived as: $W A M=\log _{10}(N E+1)$

\section{EXPERIMENTAL RESULTS AND ANALYSIS}

The goal of all quality assessment metrics is to make quality measures in agreement with subjective opinions of human observers. A data set of images whose quality has been evaluated by human subjects is required. LIVE database contains 20 original images and 779 degraded images. In this database [17], an extensive experiment was conducted to obtain scores from human subjects for a number of distorted images with different distortion types: JPEG2000, JPEG, white noise (WN), gaussian blur (GB), and bit errors in JPEG2000 bitstream when transmitted over a simulated fast-fading Rayleigh channel (FF). LIVE database has been recognized as the standard database for image quality assessment measures performance evaluation. We use two 
evaluation criteria: Pearson linear correlation coefficient (CC) and Spearman rank-order correlation coefficient (ROCC). A logistic function [9] can be used to fit the image quality assessment metrics outputs via an optimal search method to obtain their different parameters which minimize the sum squared error between the transformed image quality metrics outputs and the corresponding subjective ratings [9]. Figure 3 illustrates these results.

Table 1 summarizes the results obtained with WSSIM [18], WBCT [8], MSDD [9] and the proposed metric WAM. The obtained results confirm the prediction accuracy and robustness of the proposed image quality metric. This prominent performance owes first to the wave atoms transform, by which the loss of fidelity are analyzed in an adapted multiscale and multidirectional aspects and secondly to the take into account of HVS in the masking step.

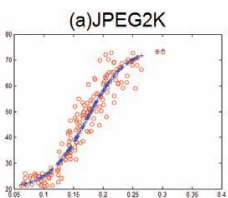

(d) $G B$

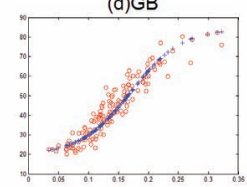

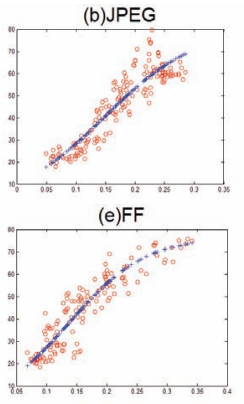

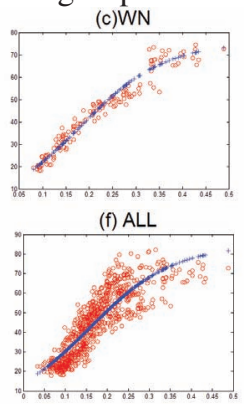

Fig3. Subjective ratings of perceived distortion for different classes of the LIVE database plotted against predicted values. In all graphs, the vertical axis denotes DMOS. The horizontal axis corresponds to the proposed metric.

\begin{tabular}{|l|l|l|l|l|l|l|}
\hline \multicolumn{7}{|c|}{ Correlation coefficient } \\
\hline & jpeg2k & Jpeg & Wn & Gb & Ff & All \\
\hline wam & 0.980 & 0.971 & 0.980 & 0.971 & 0.956 & 0.933 \\
\hline wssim & 0.940 & 0.935 & 0.962 & 0.952 & 0.953 & 0.884 \\
\hline wbct & 0.914 & 0.880 & 0.970 & 0.377 & 0.812 & 0.674 \\
\hline msdd & 0.942 & 0.940 & 0.984 & 0.959 & 0.919 & 0.890 \\
\hline \multicolumn{7}{|c|}{ Rank order correlation coefficient } \\
\hline wam & 0.979 & 0.957 & 0.988 & 0.973 & 0.956 & 0.949 \\
\hline wssim & 0.931 & 0.899 & 0.957 & 0.960 & 0.962 & 0.879 \\
\hline wbct & 0.919 & 0.825 & 0.979 & 0.312 & 0.782 & 0.624 \\
\hline msdd & 0.936 & 0.904 & 0.978 & 0.958 & 0.916 & 0.904 \\
\hline
\end{tabular}

Table 1. Correlation results with LIVE database.

\section{CONCLUSION}

This paper presents a new image quality metric based on wave atoms transform. The efficiency of the representation and analysis offered by wave atoms transform permits a very good quantization of the image distortions. It has been shown that by incorporating some properties of the HVS such as contrast masking and entropy masking we develop an efficient image quality metric. The large amount of comparison experiments proof the efficiency of the proposed image quality metric and the consistency with the human visual system.

\section{REFERENCES}

[1] Z. Wang, A. Bovik, H. Sheikh, E. Simoncelli, "Image quality assessment: from error visibility to structural similarity", IEEE Trans. Image Process. 13, 600-612, 2004.

[2] C. L. Yang, W. R. Gao and L. M . Po, "Disrecte Wavelet transform-based Structural Similarity for Image Quality Assessment", IEEE ICIP, 377-380, 2008.

[3] H.R. Sheikh, A.C. Bovik, G. de Veciana, "An information fidelity criterion for image quality assessment using natural scene statistics", IEEE Trans. Image Process. 14, 2117-2128, 2005.

[4] H.R. Sheikh, A.C. Bovik, "Image information and visual quality," IEEE Trans. Imgae Process. 15(2) 430-444, 2006.

[5] D. M. Chandler and S. S. Hemami, “ VSNR: A Wavelet-based visual signal to noise ratio for natural images", IEEE Trans. Image Process. 16(9) 2284-2298, 2007.

[6] D. H. Hubel and T. N. Wiesel, "Receptive fields, binocular interaction and functional architecture in the casts visual cortex", $J$. Physiol., 160, 106-154, 1962.

[7] J. X. Pang, R. Zhang, H. Zhang, X. Huan and Z. K. Liu, "Image quality assessment metrics by using directional projection", Chinese Optics Letters, 6 (7) 491-494, 2008.

[8] X. Gao, W. Lu and D. Tao "Wavelet based contourlet in quallity evaluation of digital images" Neurocomputing, 72 (1-3) pp, 378-385, 2008

[9] Mingna Liu and Xin Yang, "Image quality assessment using contourlet transform", optical engineering, 48(10)107201, 2009.

[10] L. Demanet, L. Ying, "Wave atom and sparsity of oscillatory patterns”, Appl. Comput, vol. 23, Issue 3, pp. 368-387, 2007.

[11] H. Führ, L. Demaret, F. Friedrich, "Beyond wavelets: New image representation Paradigms," Survey article, preprint version, 2005.

[12] E. Candes, D. Donoho, "Ridgelets: A key to higherdimensional intermittency?", Philosophical transactions Royal Society, Mathematical, physical and engineering sciences, vol. 357, no. 1760, pp.2495- 2509, 1999.

[13] E. Candes, D. Donoho, "Curvelets - A surprisingly effective nonadaptive representation for objects with edges, curves and surfaces," Curves and Surfaces, Vanderbilt University Press, Nashville, TN, 1999.

[14] A. B. Watson, "The cortex transform: rapid computation of simulated neural images", Computer Vision, Graphics, and Image Processing, vol. 39(3), pp. 311_327, Sept. 1987.

[15] A. B. Watson, R. Borthwick and M. Taylor, "Image quality and entropy masking," In Proc. SPIE, vol. 3016, 2-12, 1997.

[16] A. Ninassi, O. Le Meur, P. Le caller and D. Barba, "On the performance of human visual system based image quality asssessment metric using wavelet domain", In proc. SPIE, vol. 6806, 680610-680610-12, 2008.

[17] http://live.ece.utexas.edu/

[18] Z. Wang and EP Simoncelli. "Translation insensitive image similartity in complex wavelet domain. ICASSP, Vol. 2, pp. 573576, March 2005.

[19] A. Beghdadi, B. Pesquet-Popescu, "A new image distortion measure based on wavelet decomposition". ISSPA, vol 1, pp. 485488, 2003. 